\title{
Mediação no Zulliger: Evidências de Validade em Amostra de Não Pacientes
}

\author{
Jucelaine Bier Di Domenico Grazziotin - Universidade de Passo Fundo, Passo Fundo, Brasil \\ Silvana Alba Scortegagna - Universidade de Passo Fundo, Passo Fundo, Brasil
}

\begin{abstract}
Resumo
Considerando a influência de elementos culturais na avaliação da personalidade e a diversidade destes no cenário brasileiro, buscou-se verificar a validade do Zulliger em uma amostra gaúcha de não pacientes, focalizando as variáveis de mediação. Participaram 40 indivíduos, com idades entre 18 e 43 anos, de ambos os gêneros, escolaridade de ensino médio, do interior do Rio Grande do Sul. Foram feitas análises estatísticas descritivas e comparativas entre o grupo gaúcho e os dados normativos brasileiros. Os resultados demonstraram diferenças estatisticamente significativas entre as duas amostras no aumento das variáveis $\mathrm{XA} \%, \mathrm{WDA} \%, \mathrm{X}+\%$, no rebaixamento de $\mathrm{X}-\%$ e S- no grupo gaúcho e evidenciaram a validade do instrumento para o contexto pesquisado. O seguimento de estudos dessa natureza com amostras ampliadas e diversificadas é necessário para que se possa assegurar que as normas estabelecidas são apropriadas para a população.

Palavras-chave: avaliação psicológica, técnicas projetivas, cultura, normas, mediação
\end{abstract}

\section{Mediation in Zulliger: Evidence of Validity in Sample of Not Patients}

\begin{abstract}
Considering the influence of cultural factors in the assessment of personality and diversity of these in the Brazilian scenario, the purpose of this study was to verify the validity of Zulliger on a sample of non-patient gauchos, focusing on the mediation variables. A total of 40 individuals of both genders participated in the study - ages varying between 18 and 43 years old - all of them with high school education level, in the state of Rio Grande do Sul. Descriptive and comparative statystical analysis were used between the gaucho group and the Brazilian normative data. The results demonstrated statistically significant differences between the samples, with an increase in the variable(s) XA\%, WDA $\%, \mathrm{X}+\%$ and decrease in the variable(s) $\mathrm{X}-\%$ and $\mathrm{S}$ - in the gaucho group, ultimately showing the validity of the instrument for the context of the research. Additional and follow up studies of this nature with expanded and diverse samples is needed to ensure that the established standards are appropriate for the population.

Keywords: psychological assessment, projective techniques, culture, standards, mediation
\end{abstract}

\section{Mediación en el Zulliger: Evidencias de Validez en Muestra de No Pacientes}

\begin{abstract}
Resumen
Considerando la influencia de elementos culturales en la evaluación de la personalidad y la diversidad de estas en el escenario brasileño, se buscó verificar la validez del Zulliger en una muestra gaucha de no pacientes, enfocando en las variables de mediación. Participaron 40 individuos del interior de Rio Grande del Sur, con edades entre 18 y 43 años, de ambos sexos, con enseñanza secundaria. Fueron hechos análisis estadísticos, descriptivos y comparativos entre el grupo gaucho y los datos normativos de Brasil. Los resultados mostraron diferencias estadísticamente significativas entre las dos muestras en el aumento de las variables $\mathrm{XA} \%$, WDA $\%, \mathrm{X}+\%$, en el descenso de $\mathrm{X}-\%$ y S- en el grupo gaucho y evidenciaron la validez del instrumento en el contexto investigado. Es necesario continuar con estudios de esta naturaleza, con muestras ampliadas y diversificadas, para que se pueda garantizar que las normas establecidas son apropiadas para la población.

Palabras clave: evaluación psicológica, técnicas proyectivas, cultura, normas, mediación
\end{abstract}

O século XX marca um tempo no qual os hábitos culturais, determinados por grandes centros e potências, passam a ser adotados em escala mundial, atingindo a economia e a cultura global, com o excesso de produtos Made in the World (World Trade Organization, 2012). Apesar dessa semelhança mundial, deve-se atentar para as relações entre variáveis demográficas e as caracteristicas de comportamento dos indivíduos, dos grupos e subgrupos. As diferenças de origens culturais, étnicas, educativas, costumam incidir no desenvolvimento da personalidade, refletem-se na maneira como as pessoas percebem os estímulos e, consequentemente, podem interferir nos resultados obtidos nos testes psicológicos (Meyer, Giromini, Viglione, Reese, \& Mihura, 2015).

Em consequência, no contexto de avaliação psicológica internacional, existe preocupação em manter um padrão de excelência dos testes, sendo estes constantemente revisados e atualizados para a população em estudo (American Educational Research Association, American Psychological Association, National Council for Measurement in Education, 1999). Essa inquietação é particularmente demonstrada em pesquisas que buscam aprimorar os dados normativos dos instrumentos, que levam em conta as diferenças socioculturais da população e visam 
compreender o indivíduo e o ambiente (sociedade e cultura) em que está inserido, seus valores, tradições e hábitos transmitidos ao longo do tempo. Dessa forma, historicamente já está bem estabelecida a importância desses fatores no desenvolvimento da personalidade (Constantino, Flanagan, \& Malgady, 1995; Markus \& Kitayama, 1998; Okasaki, 1998; Ritzler, 2004).

No Brasil, apesar da qualidade dos instrumentos que os pesquisadores da área de avaliação psicológica fornecem ao mercado, existem questionamentos sobre se os aspectos demográficos estariam sendo atendidos de maneira a representar as condições socioculturais (Alchieri, 2010; Borsa, Damásio, \& Bandeira, 2012; Nascimento, 2012; Oliveira, Balarini, Marques, \& Pasian, 2011; Villemor-Amaral \& Pasqualini-Casado, 2006). O país é o quinto maior do mundo em área territorial, sendo uma terra de grandes proporções físicas e, assim, com uma convivência marcada pela diversidade. A imensa extensão do território brasileiro leva a que o país seja abrangido por diferenças climáticas, de costumes, hábitos e as mais variadas culturas. Consequentemente, em diversas regiões e sub-regiões, observam-se diferenças comportamentais, como as relativas à adequação social, relacionamento interpessoal e afetividade (Instituto Brasileiro de Geografia e Estatística, 2009; Nascimento, 2012).

Ao se considerar o contexto sociocultural que configura o Rio Grande do Sul, por exemplo, observa-se uma área tipicamente imigratória. Neto e Bezi (2008) elucidam que o estado apresenta um mosaico cultural expresso pela presença de distintas etnias (italiana, alemã, polonesas, mistas), embora o gaúcho típico possua traços particulares observados no conservadorismo, no uso de bombacha, do chapéu e do pala, nas danças tradicionalistas, na participação em Centros de Tradições Gauchescas (CTGS), na apropriação da gastronomia nativa, como o churrasco e o chimarrão.

A adaptação social, o relacionamento interpessoal e a afetividade estão entre as características de pessoas que fazem parte da população gaúcha (Di Domenico Grazziotin \& Scortegagna, 2012; 2013). No contexto geral, pode-se dizer que esse povo se identifica mediante simbologias materializadas via costumes e tradições, que remetem a um modo de vida singular se comparados a outras regiões do Brasil.

Já, o estado de São Paulo, agrega pessoas de todas as regiões do país, há uma maior heterogeneidade e diversidade sociocultural. Verifica-se uma intensa proximidade com a vida urbana, com o desenvolvimento de tecnologias, centros de pesquisa, indústrias de grande porte e telecomunicações, e um incremento ao acesso à cultura (teatros, museus, cinemas, feiras e eventos científicos). A busca por inovação, a criatividade, a ousadia, o empreendedorismo são características observadas nas pessoas que habitam esse estado (Ferreira \& Villemor Amaral, 2005; Instituto Brasileiro de Geografia e Estatística, 2009; Nascimento, 2012).

Dessa forma, estudos e atualizações dos instrumentos de que se dispõe são necessários para que se estabeleçam normas adaptadas à cultura do meio imediato e sensíveis às transformações que ocorrem. Pesquisas de normatização são, ainda, essenciais para que as interpretações sejam fidedignas ao contexto, supondo-se que diferenças de comportamentos determinadas pela cultura podem, eventualmente, alterar os desempenhos esperados (Okasaki, 1998; Ritzler, 2004). De tal modo, pode haver circunstâncias nas quais o resultado do teste deve ser avaliado em relação às respostas normativas de um subgrupo de pessoas específico, que possua características demográficas semelhantes à do indivíduo (Okasaki, 1998; Weiner, 2000).

$\mathrm{Na}$ maior parte das vezes, as diferenças são verificadas com a utilização dos testes objetivos, porém os métodos projetivos também apresentam sensibilidade à cultura (Constantino et al., 1995). Ainda que o método dos borrões de tinta, por exemplo, ofereça extensa probabilidade de administração independentemente da cultura, isso não o isenta da possibilidade de obter resultados distintos quando conduzido em diferentes populações (Ritzler, 2004; Weiner, 2000).

No caso do Zulliger no Sistema Compreensivo (ZSC), algumas investigações avaliaram em que medida é possível extrapolar a aplicabilidade do Sistema Compreensivo do Rorschach e utilizar parâmetros normativos adaptados a diferentes populações (Fazendeiro \& Novo, 2012; Franco \& Villemor-Amaral, 2012a; Mattlar et al., 1990; Vilches \& Olivos, 2004; Villemor-Amaral \& Cardoso, 2012; Zdunic, 2003; 2011) e em diversos contextos (Di Domenico-Grazziotin \& Scortegagna, 2012; 2013; Franco \& Villemor-Amaral, 2012b). $\mathrm{O}$ instrumento mostra-se eficiente na avaliação do funcionamento psíquico, com base nos agrupamentos de variáveis que compõem aspectos cognitivos e psicodinâmicos da personalidade (Villemor-Amaral \& Primi, 2012; Zdunic, 2003).

Dentre os agrupamentos, destaca-se o das variáveis de mediação, que inclui: respostas adequadas com o mínimo de distorções subjetivas (XA\%), respostas apropriadas em que a localização é W ou D (WDA\%), respostas de qualidade formal convencional $(\mathrm{X}+\%)$, 
de qualidade formal inusual $(\mathrm{Xu} \%)$, de qualidade formal distorcida (X-\%), respostas de espaço branco com qualidade formal negativa $(S-)$, respostas populares $(P)$. Esse módulo revela tanto o funcionamento cognitivo quanto o grau de adaptabilidade e adequação do indivíduo em relação ao meio onde vive, representando um dos aspectos mais importantes de sua personalidade (Exner, 2003; Weiner, 2000).

Tais variáveis estão associadas ao processo de identificação e tradução da informação. Esse processo ocorre a partir dos estímulos do teste e, assim, o mundo representacional medeia o contato com a realidade. A análise da mediação permite formular hipóteses sobre se o indivíduo vê e entende a informação que recebe como a maior parte das pessoas, se ele se afasta do que é convencional ou não (Zdunic, 2003).

No entanto, ao mesmo tempo em que há algumas variáveis mais constantes, que não se modificam de acordo com a condição sociocultural, a etnia e a nacionalidade do indivíduo, há outras que são mais suscetíveis a alterações (Weiner, 2000). Segundo o autor, as respostas $\mathrm{X}_{-}^{-} \%$, que se referem a formas não realistas de perceber o mundo e exibem aspectos constantes da personalidade, permanecem inalteradas, independentemente da cultura. Outras, são mais vulneráveis às diferenças culturais e, dependendo da frequência com que são fornecidas, podem conduzir a interpretações equivocadas, como no caso de $\mathrm{P}, \mathrm{X}+\%$, e $\mathrm{Xu} \%$.

Um estudo importante, desenvolvido por Nascimento (2012), buscou ampliar a amostra de referência do estudo normativo do Rorschach no Sistema Compreensivo para o Brasil. A amostra foi composta de 209 protocolos de adultos, não pacientes, de cidades do interior e do litoral, do estado de São Paulo. Os adultos da capital apresentaram respostas menos convencionais, valores significativamente mais baixos em XA\%, WDA $\%, X^{+\%} \%$ e mais altos em X-\%, WSum6, Sum H, em comparação com os adultos de cidades do interior, que demonstraram mais predisposição para um bom relacionamento interpessoal, com menor frequência de PHR. A autora considera a necessidade de se estudar cada população, com seu modo peculiar de responder aos testes psicológicos e dar continuidade a trabalhos como este, em outras regiões.

Outro estudo interessante desenvolvido por Meyer, Giromini, Viglione, Reese e Mihura (2015) examinou a associação entre gênero, etnia, idade e educação, com os escores do Rorschach. Os autores incluiram na amostra os dados do R-PAS de indivíduos provindos de 13 países $(n=640)$ e uma amostra clínica $(n=490)$ de pessoas, que responderam ao sistema compreensivo. Entre os resultados, a variável PER foi significativamente mais elevada em indivíduos de cor branca, comparativamente aos de cor negra e entre hispânicos, asiáticos e aqueles com misturas de raças. $\mathrm{Na}$ amostra clínica de jovens, o aumento da idade foi associado com percepção mais convencional e processos de pensamento menos ilógicos, e na normativa de adultos, as variáveis $T$ e PER aumentaram com a idade. A escolaridade demonstrou associação com a síntese cognitiva e os recursos de enfrentamento.

Diante da relevância dessas complexidades, faz-se necessário verificar os estudos normativos e transculturais com o ZSC. Internacionalmente, as pesquisas foram conduzidas na Finlândia (Mattlar et al., 1990; Mattlar et al., 1993), no Chile, (Vilches \& Olivos, 2004), na Argentina (Zdunic, 2011), no Peru (Carpio \& Lugón, 2011) e em Portugal (Fazendeiro \& Novo, 2012). Os resultados mais recentes indicaram valores normativos atualizados para as variáveis $\mathrm{R}, \mathrm{L}, \mathrm{EA}$, es, $\mathrm{P}, \mathrm{X}-\mathrm{\%}$, WSum6 (Zdunic, 2011), a necessidade de investigações mais intensas sobre o sentido interpretativo do teste (Carpio \& Lugón, 2011; Fazendeiro \& Novo, 2012), e do seguimento de estudos dessa natureza.

No Brasil, a pesquisa de referência normativa com o ZSC foi conduzida por Franco, Cardoso, Villemor-Amaral e Primi (2012) e resultou na elaboração de um manual para o teste. A amostra normativa de não pacientes, procedente de instituições do interior do mesmo estado (uma instituição de ensino e quatro empresas comerciais), foi subdividida em dois grupos: uma com escolaridade de nível superior e a outra com escolaridade de ensino fundamental (14,5\% fundamental I, $12,2 \%$ fundamental II) e ensino médio (28,6\%).

Outro estudo normativo buscou evidências de validade do tipo de vivência (EB) no ZSC, por meio de correlações com o indicador (EB) do Rorschach (Villemor-Amaral \& Cardoso, 2012). Nas correlações entre o número de respostas $M$ e o escore WSumC, verificou-se que o Zulliger e o Rorschach produzem respostas $M$ de modo mais semelhante do que as respostas de cor. A correspondência também foi observada entre os participantes considerados introversivos, extratensivos e evitativos no Rorschach-SC e no Zulliger. Entretanto, houve discordâncias no tipo ambigual. Essa fragilidade sugere a necessidade de se estabelecer novas proporções entre M e WSumC no Zulliger, para definir o tipo de vivência, e de se alterar as instruções do teste no sentido de motivar a emissão de um número maior de respostas em cada prancha. 
$\mathrm{Na}$ perspectiva de fazer adequações aos estudos normativos, Franco e Villemor-Amaral (2012a) verificaram se as constelações e as variáveis que informam sobre possíveis distúrbios e disfunções psíquicas, utilizadas no método de Rorschach-SC, aplicam-se ao ZSC. A comparação entre a média dos grupos evidenciou resultados parcialmente positivos para as psicopatologias da esquizofrenia e depressão.

Alguns estudos interessantes abordam variáveis do ZSC com perspectivas de entender o comportamento de indivíduos e o contexto em que estão inseridos (Di Domenico-Grazziotin \& Scortegagna, 2012; 2013; Ferreira \& Villemor-Amaral, 2005; Franco \& Villemor-Amaral, 2012b). Na região sudeste, Ferreira e Villemor-Amaral (2005) buscaram a correlação de algumas variáveis do ZSC, entre as quais, $\mathrm{X}+\%, \mathrm{D} \%, \mathrm{Dd} \%$, DQV\%, Xu\%, S, Na, Xy, FM, SumT, SumM, SumY, $\mathrm{EB}$, Art, A, Fi, com um questionário de avaliação de desempenho de uma empresa. As correlações foram positivas e indicaram uma predição do bom desempenho dos profissionais: $\mathrm{X}+\%$ correlacionou-se com solução de problemas; Dd correlacionou-se negativamente com a capacidade para solucionar problemas; e o aumento de $Y$ demonstrou a presença de pessoas com elevado estresse situacional e características depressivas.

Franco e Villemor-Amaral (2012b) investigaram a validade incremental do teste de ZSC e do Pfister, a partir da avaliação da personalidade de brasileiros adictos de álcool e de franceses dependentes da heroína. Os resultados do ZSC indicaram dificuldade para seguir e respeitar as regras sociais, o rebaixamento das respostas populares $\mathrm{P}$ do determinante de forma $(\mathrm{F} \%)$. Os protocolos das pessoas que não reagiram bem ao tratamento da desintoxicação da droga evidenciaram sinais de uma personalidade mais desconectada da realidade, e a presença de $\mathrm{X}$ - nos usuários brasileiros. Os resultados ratificaram as informações geradas pelos instrumentos.

$\mathrm{Na}$ região sul, Di Domenico-Grazziotin e Scortegagna $(2012$; 2013) realizaram estudos com a finalidade de buscar evidências de validade para o ZSC. Todas as variáveis do agrupamento Relacionamento interpessoal permaneceram nos parâmetros normativos, exceto COP e Sum T que ficaram acima da média. Os participantes demonstraram boa capacidade de relacionamento interpessoal e habilidade social e foram obtidas correlações significativas entre as variáveis do ZSC e os fatores do IHS-Del Prette.

Observa-se que a maior parte das amostras das pesquisas referidas concentrara-se na região sudeste, o que pode se apresentar como uma limitação na confiabilidade das interpretações propostas, quando se consideram o contexto nacional e sua diversidade. Diante dessa constatação, constitui-se objetivo deste estudo verificar evidências de validade do teste de Zulliger em uma amostra gaúcha de não pacientes, focalizando as variáveis de mediação.

A escolha desse agrupamento de variáveis deu-se por muitas razões: i) segundo Weiner (2000), apenas uma pequena parte do esquema de codificação do sistema compreensivo como os códigos de conteúdo, especialmente as respostas populares, qualidade formal ordinárias e incomuns e a localização podem, potencialmente, serem influenciadas por diferenças culturais; ii) trata-se de estudo exploratório, fruto de uma pesquisa mais extensa que inclui outros agrupamentos; iii) a amostra é composta de não pacientes e as variáveis de mediação denotam ser as mais apropriadas para revelarem o grau de adaptabilidade, de convencionalidade ao meio; iv) são escassas as pesquisas sobre as diferenças socioculturais entre indivíduos de distintos estados. Considerando o exposto, espera-se que ocorram diferenças estatisticamente significativas entre o grupo gaúcho e os dados normativos nacionais nas variáveis de mediação por estarem situados em diferentes regiões brasileiras, e o ZSC ser sensível para captar tais diferenças.

\section{Método}

Participaram desse estudo 40 profissionais do comércio, entre 18 e 43 anos $(M=26,5 ; D P=7,99)$, sendo $23(57 \%)$ do gênero feminino e 17 (43\%) do gênero masculino, 34 (85\%) com ensino médio completo e $6(15 \%)$ com ensino fundamental II, $(M=$ 10,40; $D P=1,24)$, escolhidos intencionalmente. Os participantes foram provenientes de duas lojas de supermercados de uma cidade do interior do estado do Rio Grande do Sul, com tradição da cultura gaúcha e predomínio da agricultura. Todos os participantes já haviam realizado avaliações psicológicas e médicas para serem admitidos na empresa, tendo sido contratados por apresentarem um perfil condizente com as atribuições de vendas e atendimento ao público, constituindo-se em uma amostra menos diversificada. Consistiram critérios de exclusão a ausência de história de tratamento psiquiátrico e problemas auditivos ou visuais que impossibilitassem a execução do teste.

\section{Instrumentos}

Entrevista semiestruturada. Constituiu-se de um protocolo com informações sobre idade, gênero, 
estado civil, escolaridade, nível socioeconômico, ocupação, condições familiares e de saúde. As questões foram elaboradas pelas pesquisadoras com o objetivo de obter dados sociodemográficos e ratificar os critérios de inclusão ou exclusão da amostra.

Teste Zulliger no Sistema Compreensivo ZSC - forma individual (Villemor-Amaral \& Primi, 2012) - é uma técnica de avaliação da personalidade, composta de um jogo de três cartões com manchas de tinta simétricas e diferentes em cada um deles. Considerando o objetivo proposto, foram elencadas as variáveis que compõem o agrupamento da mediação: respostas adequadas com o mínimo de distorções subjetivas (XA\%); respostas adequadas em que a localização é W ou $\mathrm{D}$ (WDA\%); respostas de qualidade formal convencional $(\mathrm{X}+\%)$; respostas de qualidade formal inusual $\left(\mathrm{Xu}^{\%} \%\right.$; respostas de qualidade formal distorcida (X-\%); respostas de espaço branco com qualidade formal negativa (S-); respostas populares $(\mathrm{P})$.

\section{Procedimentos}

Após a aprovação do estudo pelo Comitê de Ética em Pesquisa da Universidade de Passo Fundo (CEP/ UPF), sob o protocolo de número 076/2009, iniciou-se a coleta de dados. O contato com o responsável pelo departamento de pessoal possibilitou a indicação dos funcionários que trabalhavam diretamente com o público e que, pelas normas da empresa, já haviam sido avaliados por ocasião de terem sido selecionados para o cargo disponível. Os funcionários que aceitaram participar do estudo realizaram a entrevista, e os que preencheram os critérios de inclusão assinaram o
Termo de Consentimento Livre e Esclarecido. A partir de então, seguiu-se com a administração do ZSC nas dependências da empresa.

As codificações dos protocolos do ZSC foram realizadas pela pesquisadora responsável pelo estudo e discutidas pelas autoras deste artigo. Para maior confiabilidade dos dados, efetuou-se um estudo de fidedignidade, a partir da recodificação de $25 \%$ dos protocolos por um juiz independente e procedeu-se à análise do coeficiente Kappa, que revelou valores acima de 0,80 para todas as variáveis do Zulliger. Os resultados foram obtidos por meio de procedimentos estatísticos no SPSS, utilizando-se a análise descritiva e o $t$ de Student para a comparação do desempenho entre os grupos. Foi adotado como nível de significância um valor de $p<0,05$. Os dados foram confrontados com as expectativas normativas de não pacientes (Villemor-Amaral \& Primi, 2012, p. 122).

\section{Resultados}

Apresentam-se os resultados das estatísticas descritivas do ZSC para as variáveis do módulo de mediação da amostra gaúcha e dos valores de referência nacional. As diferenças estatisticamente significativas exibidas pelos dois grupos por meio do teste $t$ de Student, e a magnitude de tais diferenças, alcançadas por meio do $d$ de Cohen, podem ser observados na Tabela 1 .

Os dados expostos na Tabela 1 permitem observar que a média, o desvio padrão, o teste $t$ de Student e o $d$ de Cohen, indicam que, das sete variáveis do módulo da mediação na amostra gaúcha, cinco se diferenciaram

Tabela 1

Comparação do Desempenho dos Grupos em Relação às Variáveis de Mediação do ZSC

\begin{tabular}{lcccccccccccccc}
\hline & \multicolumn{1}{c}{ Amostra gaúcha $(N=40)$} \\
\hline Variáveis & M & DP & Min. & Máx. & P5 & P25 & P50 & P75 & P95 & M & DP & t & d & p \\
XA $\%$ & 83,8 & 11,1 & 55 & 100 & 57,7 & 77 & 83,5 & 89,5 & 100 & 73 & 21 & 6,163 & $-0,55$ & $0,001^{*}$ \\
WDA $\%$ & 73,8 & 15,1 & 38 & 100 & 50 & 63,7 & 71 & 85 & 100 & 65,9 & 20,2 & 3,301 & $-0,41$ & $0,002^{*}$ \\
X-\% & 15,3 & 11,3 & 0 & 44 & 0 & 8,5 & 14,5 & 22 & 41,4 & 21 & 15,9 & $-3,21$ & 0,37 & $0,003^{*}$ \\
X+ \% & 54,8 & 16,5 & 22 & 85 & 23,2 & 44 & 56 & 69 & 84,9 & 47,2 & 21,3 & 2,909 & $-0,37$ & $0,006^{*}$ \\
Xu \% & 28,3 & 15,4 & 0 & 55 & 0 & 14,5 & 28,5 & 40,7 & 50 & 25,7 & 17,8 & 1,048 & $-0,15$ & 0,301 \\
S- & 0,2 & 0,4 & 0 & 1 & 0 & 0 & 0 & 0 & 1 & 0,3 & 0,5 & $-2,62$ & 0,21 & $0,012^{* *}$ \\
P & 1,6 & 1,0 & 0 & 5 & 0 & 0 & 1,5 & 2 & 3,95 & 1,4 & 1,1 & 1,288 & $-0,18$ & 0,205 \\
\hline
\end{tabular}

* teste $t$ de Student ao nível de $1 \%$.

** teste $t$ de Student ao nível de $5 \%$.

Psico-USF, Bragança Paulista, v. 21, n. 1, p. 63-72, jan./abr. 2016 
significativamente dos indicadores de referência nacional, sendo que três mostraram-se acima dos parâmetros normativos: $\mathrm{XA} \%(M=83,78 ; D P=11,06 ; p=0,001$; $d=-0,55) ; \mathrm{WDA} \%(M=73,78 ; D P=15,09 ; p=0,002$; $d=-0,41) ; \mathrm{X}+\%(M=54,78 ; D P=16,47 ; p=0,006 ; d$ $=-0,37)$, e duas revelaram-se abaixo da média: $\mathrm{X}-\%(M$ $=15,28 ; D P=11,27 ; p=0,003 ; d=0,37) ; \mathrm{S}-(M=0,2$; $D P=0,4 ; p=0,012 ; d=0,21)$. Apenas duas variáveis não se diferenciaram significativamente dos indicadores de referência nacional e mantiveram-se nos parâmetros normativos: $\mathrm{Xu} \%(M=28,25 ; D P=15,39 ; p=0,301 ; d$ $=-0,15) ; P(M=1,60 ; D P=0,98 ; p=0,205 ; d=-0,18)$.

Os resultados fazem alusão à expectativa deste estudo, de que poderia ocorrer uma diferença significativa entre as duas amostras em razão das questões socioculturais (Constantino et al., 1995; Markus \& Kitayama, 1998; Mattlar et al., 1990; Mattlar et al., 1993; Nascimento, 2012; Okasaki, 1998; Ritzler, 2004; Weiner, 2000). Contudo, outros fatores relacionados às particularidades da amostra (idade, atividade profissional, indivíduos procedentes de cidade do interior) podem ter contribuído para esses achados (Nascimento, 2012; Neto \& Bezi, 2008).

\section{Discussão}

Este estudo demonstrou diferenças significativas nas variáveis do agrupamento da mediação do teste de ZSC entre amostra gaúcha de não pacientes, em comparação com os dados de referência nacional, conferindo evidências de validade ao instrumento. A amostra da região sul demonstrou qualidades de adaptação social, ajustamento à realidade, objetividade e conservadorismo observado pelo aumento de XA\%, WDA\% e $\mathrm{X}+\%$, bem como na diminuição de $\mathrm{X}-\%$ e $\mathrm{S}-$, quando comparada com a amostra normativa.

Ainda que o mundo esteja globalizado, não se pode desconsiderar que cada região apresenta suas especificidades, suas características e sua tradição (Instituto Brasileiro de Geografia e Estatística, 2009; Neto \& Bezzi, 2008). Tais diferenças podem influenciar na dinâmica do funcionamento dos indivíduos (Alchieri, 2010; Borsa et al. 2012; Constantino et al., 1995; Markus \& Kitayama, 1998; Nascimento, 2012; Okasaki, 1998; Oliveira et al., 2011; Ritzler, 2004; Villemor-Amaral \& Pasqualini-Casado, 2006) e, por isso, estudos de normatização com o ZSC são desenvolvidos em diversos lugares do mundo (Fazendeiro \& Novo, 2012; Mattlar et al., 1990; Mattlar et al., 1993; Vilches \& Olivos, 2004; Zdunic, 2011, 2003), inclusive no Brasil (Franco et al.,
2012; Franco \& Villemor-Amaral, 2012a; Villemor-Amaral \& Cardoso, 2012).

A inquietação com a dinâmica de comportamentos e as especificidades de cada indivíduo é demonstrada em estudos que abordam as variáveis e os agrupamentos do ZSC (Di Domenico-Grazziotin \& Scortegagna, 2012; 2013; Ferreira \& Villemor Amaral, 2005; Franco \& Villemor-Amaral, 2012b). No entanto, pouco se sabe sobre as características de pessoas de outras regiões do Brasil, por exemplo, da população gaúcha (Di Domenico-Grazziotin \& Scortegagna, 2012; 2013), e também não se tem conhecimento da existência de estudos que focalizem as variáveis de mediação.

As sete variáveis do módulo da mediação (XA\%, $\mathrm{X}+\%, \mathrm{Xu} \%, \mathrm{X}-\%, \mathrm{WDA} \%, \mathrm{~S}-\%$ e $\mathrm{P})$ indicam em que medida a pessoa emite respostas convencionais e aceitáveis no que se refere à percepção da realidade e ao modo como reage ao ambiente (Villemor-Amaral \& Primi, 2012), um importante indicador na avaliação da saúde mental. Dessa forma, é interessante discutir os resultados significativos obtidos na comparação entre as duas regiões.

A variável $\mathrm{XA} \%$, quando nos valores da média normativa, denota que o indivíduo percebe os estímulos como a maioria das pessoas, fazendo um adequado uso do critério da realidade (Zdunic, 2003). Na comparação dos indicadores, a amostra gaúcha apresentou escores significativamente mais elevados do que o grupo normativo $(p=0,001 ; d=-0,55)$. A variável WDA\% que revela a capacidade de perceber os fatos de modo adequado e indica em que proporção a mediação é apropriada em situações óbvias (Villemor-Amaral \& Primi, 2012; Zudnic, 2003) apresentou um aumento estatisticamente significativo no grupo gaúcho $(\phi=$ $0,002 ; d=-0,41)$.

Esses primeiros achados das variáveis $\mathrm{XA} \%$ e WDA\% corroboraram algumas características da amostra gaúcha, de adaptação social, de percepção da realidade em um estilo mais habitual, uma tendência ao senso comum (Di Domenico-Grazziotin, \& Scortegagna, 2012; 2013; Neto \& Bezi, 2008). A expressividade da cultura gaúcha ocorre mediante inúmeros códigos, mas deve-se ater, segundo Neto e Bezi (2008), para a imaterialidade, que se manifesta por meio das crenças e dos valores mais significativos, como: respeito ao próximo, a família, ao apego a terra, a linguagem regional, ao lugar em que vive e ao tradicionalismo, além do nativismo. Essas particularidades distinguem os gaúchos da amostra normativa, que parece indicar pessoas menos 
conservadoras e que buscam por inovação (Ferreira \& Villemor Amaral, 2005; Nascimento, 2012).

Esses resultados são ratificados pelos escores da variável $X+\%$ que, quando elevados, sugerem a presença de perfeccionismo e objetividade exacerbados e, quando diminuídos, faz saber que o indivíduo é pouco convencional em suas percepções (Ferreira \& Villemor-Amaral, 2005; Villemor-Amaral \& Primi, 2012). Observa-se que, na amostra gaúcha, $\mathrm{X}+\%$ manteve-se significativamente acima da tabela normativa $(p=$ $0,006 ; d=-0,37)$.

$\mathrm{O}$ aumento da variável $\mathrm{X}+\%$ parece representar características peculiares da amostra do interior gaúcho, que se refletem nos hábitos da cultura riograndense. Os fortes costumes e as atitudes voltadas aos valores e crenças que orientam as suas ações concretizadas no espaço, originam formas típicas, passíveis de reconhecimento pelos demais grupos sociais (vestimentas típicas, danças tradicionalistas, churrasco, chimarrão) (Instituto Brasileiro de Geografia e Estatística, 2009; Neto \& Bezi, 2008). A música, por exemplo, expressa a identificação do gaúcho com o espaço em que habita, retrata o Rio Grande, enfatiza os aspectos físico-naturais e sociais. Essa expressividade denota uma devoção considerada uma forma de cultuar as tradições, e promulgar o sentimento de identificação pela terra e pela cultura (Neto \& Bezi, 2008).

Diferentemente, indivíduos do estado de São Paulo, especialmente da capital, tendem a demonstrar mais ousadia e menos tradicionalismo. $\mathrm{O}$ acesso às áreas de desenvolvimento econômico, tecnológico, cultural, educacional, de saúde e, possivelmente, a capacidade criadora, inventiva, empreendedora, progressista, seja bastante motivada (Ferreira, 2005; Nascimento, 2012). Esse achado corrobora Nascimento (2012) que também encontrou resultados significativamente mais elevados em XA\% em pessoas de cidades do interior, quando comparadas às da capital, indicando menos convencionalidade nas da capital.

Outros resultados relevantes a serem interpretados, na comparação entre os dois grupos, são as variáveis $\mathrm{X}-\%$ e S- . As respostas de qualidade formal distorcida são um bom indicador para avaliar se a pessoa percebe suas experiências de modo realista (Weiner, 2000). No grupo gaúcho, as duas variáveis mostraram-se significativamente abaixo dos parâmetros normativos: X-\% $(\phi=0,003 ; d=0,37)$ e S- $(p=0,012 ; d=0,21)$. A reduzida incidência de respostas distorcidas não denotaram interferências emocionais e afetos contraproducentes (Exner, 2003; Villemor-Amaral \& Primi, 2012).
O rebaixamento das variáveis $\mathrm{X}-\%$ e $\mathrm{S}$ - demonstram estilos e traços de comportamento da amostra gaúcha, como capacidade de adaptação ao ambiente e facilidade de comunicação com os demais. Esses resultados apoiam os achados de XA\%, WDA\%, X+\% e aventam a possibilidade da discrepância ser decorrente tanto das questões regionais quanto das características da amostra. Eles vêm ao encontro de pesquisas que mostram características afetivas em pessoas que trabalham com atendimento ao público, residentes no interior da região sul (Di Domenico-Grazziotin \& Scortegagna, 2012, 2013), e de estudo normativo que verificou menos distorção da realidade em indivíduos do interior de São Paulo, quando comparados aos da capital (Nascimento, 2012).

Considerando que a amostra gaúcha é composta de não pacientes, avaliados por médicos e psicólogos para ingressarem na empresa, os dados legitimam o estudo de Franco e Villemor-Amaral (2012b) que observaram a presença de $\mathrm{X}$ - em protocolos de diversas patologias, como a esquizofrenia e o uso de drogas. Outras qualidades da amostra gaúcha, como ter habilidades para exercerem atividades comerciais e de atendimento ao público, capacidade de adaptação ao ambiente, podem ter contribuído nesses achados. Essa comprovação corrobora a ideia das respostas $\mathrm{X}-\%$ exibirem aspectos constantes da personalidade, independentemente da cultura (Weiner, 2000).

$\mathrm{Na}$ amostra estudada, houve algumas diferenças na comparação com a normativa que podem ser atribuídas às especificidades da cultura riograndense. Alguns autores (Constantino et al., 1995; Markus \& Kitayama, 1998; Okasaki, 1998; Nascimento, 2012; Ritzler, 2004) referem estar suficientemente instituído o valor desse fator na construção da personalidade. Portanto, a interferência de questões culturais da região sul pode ter incidido sobre os resultados deste estudo, especialmente por ser uma amostra de cidade do interior, de pessoas que cultivam fortemente a tradição, que mantêm hábitos e costumes relativos ao meio em que vivem.

Indo um pouco mais além, torna-se importante considerar que esses resultados se referem a um agrupamento específico de variáveis e, embora os participantes desta pesquisa tenham certa semelhança com a amostra normativa brasileira (gênero, escolaridade, profissão, não pacientes), eles apresentam particularidades que levam a ter cautela na compreensão dos resultados e a impedir que sejam generalizados. Destaca-se o número reduzido da amostra, o seu caráter homogêneo, o fato de ser composta de pessoas que 
ocupam funções de atendimento ao público, que realizaram avaliação médica e psicológica para o ingresso da atividade empresarial, o que lhes confere um suposto caráter mais sadio. A amostra gaúcha constituiu-se de um grupo menos expressivo de pessoas $(N=40)$ de até 43 anos $(D P=7,99)$, procedentes de duas lojas de supermercados de uma cidade do interior do estado do RS; enquanto a normativa é mais extensa $(N=220)$, a idade atingiu os 83 anos $(D P=12,2)$, e foi realizada com pessoas de empresas diversificadas (transporte, aviário, telecomunicações e comercial), oriundas de cidades da capital do estado de São Paulo e do interior.

A importância de se examinar a associação entre as variáveis demográficas, como gênero, idade, escolaridade, atividades de trabalho e os resultados dos testes psicológicos é salientada em estudos do Rorschach (Constantino et al., 1995; Meyer et al., 2015; Nascimento, 2012; Ritzler, 2004) e do ZSC (Carpio \& Lugón, 2011; Fazendeiro \& Novo, 2012; Franco \& Villemor-Amaral, 2012b; Vilches \& Olivos, 2004; Zdunic, 2003, 2011), pois podem impactar nos resultados atribuídos à etnia e às diferenças culturais. Outras questões, como nível socioeconômico e incidência de patologias também devem ser consideradas (Meyer et al., 2015, Okasaki, 1998; Ritzler, 2004).

Os resultados deste estudo apoiam investigações prévias que demonstraram as evidências de validade do Zulliger, incrementam o poder do instrumento para a população estudada e fortalecem a utilização do sistema compreensivo. Todavia, impõem o desafio de se continuar pesquisando, diante das peculiaridades encontradas nos estudos realizados com essa população, para que os dados normativos possam direcionar com segurança as tendências interpretativas. Tais estudos devem contemplar amostras mais representativas e diversificadas, procedentes de regiões brasileiras variadas e controlar variáveis moderadoras que possam interferir nos resultados.

\section{Referências}

American Educational Research Association, American Psychological Association, \& National Council for measurement in Education. (1999). Standards for educational and psychological testing. Washington, DC: American Educational Research Association.

Alchieri, J. C. (2010). Análise dos dados demográficos das normas brasileiras de instrumentos psicológicos empregados na avaliação da personalidade. Revista Psicologia e Saúde, 2(1), 56-63. Recuperado de http://www.gpec.ucdb.br/pssa/index.php/pssa/ article/view/36

Borsa, J. C., Damásio, B. F., \& Bandeira, D. R. (2012). Adaptação e validação de instrumentos psicológicos entre culturas: Algumas considerações. Paidéia, 22(53), 423-432. doi: 10.1590/1982-43272253201314.

Carpio, R. S., \& Lugón, C. M. (2011). Validación del sistema comprehensivo de Exner en el test de Zulliger. Persona, 14, 145-158. Recuperado de http:// www.redalyc.org/articulo.oa?id=147122650006

Constantino, G., Flanagan, R., \& Malgady, R. (1995). The history of the Rorschach: Overcoming bias in multicultural projective assessment. Rorschachiana: Yearbook of the International Rorschach Society, 20,148171. Recuperado de http://psycnet.apa.org/index. cfm? fa=buy.option ToBuy\&id $=2011-21143-010$

Di Domenico-Grazziotin, J. B., \& Scortegagna, S. A. (2012). Zulliger e habilidade social: Evidências de validade no contexto empresarial. Psicologia: Reflexão e Crítica, 25(1), 69-78. doi: 10.1590/S0102-79722012000100009.

Di Domenico-Grazziotin, J. B., \& Scortegagna, S. A. (2013). Relacionamento interpessoal, produtividade e habilidades sociais: Um estudo correlacional. Psico-USF, 18(3), 491-500. doi: 10. 1590/ S14138271201300 0300015

Exner, J. E. (2003). The Rorschach: A comprehensive system. vol. I: Basic foundations. New York: Wiley \& Sons.

Fazendeiro, H. M. M., \& Novo, R. de J. F. (2012). Versão coletiva do teste de Zulliger segundo o sistema compreensivo de Rorschach. Avaliação Psicológica, 11(3), 407-422. Recuperado de http:/ / pepsic.bvsalud.org/pdf/avp/v11n3/v11n3a08.pdf

Ferreira, M. E. A., \& Villemor-Amaral, A. E. (2005). $O$ teste de Zulliger e avaliação de desempenho. Paidéia, 15(32), 367-376. doi: 10.1590/ S0103-863X2005000300006

Franco, R. R. C, Cardoso, L. M., Villemor-Amaral, A. E., \& Primi, R. (2012). Estudos de normatização, precisão e validade. Em A. E. Villemor-Amaral \& R. Primi (Eds.). Teste de Zulliger no sistema Compreensivo ZSC: Forma individual (pp. 115-130). São Paulo: Casa do Psicólogo.

Franco, R. R. C., \& Villemor-Amaral, A. E. (2012a). O Zulliger e as constelações do Rorschach no Sistema 
Compreensivo. Avaliação Psicológica, 11(1), 141-152. Recuperado de http://pepsic.bvsalud.org/pdf/ avp/v11n1/v11n1a13.pdf

Franco, R. R. C., \& Villemor-Amaral, A. E. (2012b). Validade incremental do Zulliger e do Pfister no contexto da toxicomania. Psico-USF, 17 (1), 73-83. doi: 10.1590/S1413-82712012000100009

Instituto Brasileiro de Geografia e Estatística (2009). Indicadores sociodemográficos e de saúde no Brasil: 2009. Rio de Janeiro: Estudos \& Pesquisas IBGE.

Markus, H. R., \& Kitayama, S. (1998). The cultural psychology of personality. Journal of Cross-Cultural Psychology, 29(1), 63-87. doi: 10.1177/0022022198291004

Mattlar, C. E., Forsander, C., Norrlund, L., Carlson, A., Vesala, P., Oist, A. E., \& Uhinki A. (1993). A Zulliger workbook for appling the Rorschach comprehensive system. Turku (Finlãndia): The Research and Development Unit of the Social Insurance Institution Mimeo.

Mattlar, C. E., Sandahl, C., Lindeberg, S., Lehtinen,V., Carlsson, A., Vesala, P., \& Mahmmod, Z. (1990). Methodological issues associated with the aplication of the comprehensive system when analyzing the Zulliger, and the structural resemblance between the Zulliger and Rorschach. British Journal of Projective Psychology, 35(2), 17-27.

Meyer, G. J., Giromini, L., Viglione, D. J., Reese J. B., \& Mihura J. L. (2015). The Association of Gender, Ethnicity, Age, and Education With Rorschach Scores. Assessment, 22 (1) 46-64. doi: 10.1177/1073191114544358.

Nascimento, R. S. G. F. (2012). Estudo normativo para o sistema compreensivo do Rorschach. Avaliação Psicológica, 11(3), 361-374. Recuperado de http://pepsic. bvsalud.org/pdf/avp/v11n3/v11n3a05.pdf

Neto, H. B., \& Bezi, M. L. (2008). Regiões culturais: A construção de identidades culturais no Rio Grande do Sul e sua manifestação na paisagem gaúcha. Sociedade \& Natureza, Uberlândia, 20(2), 135-155 Recuperado de http://www.scielo.br/pdf/sn/ v20n2/a09v20n2.pdf

Okasaki, S. (1998). Psychological assessment of Asian Americans: Research agenda for cultural competency. Journal of Personality Assessment, 70(1), 54-70. doi:10.1207/s15327752jpa70014
Oliveira, A. B. M., Balarini, F. B., Marques, L. A. da S., \& Pasian, S. R. (2011). Adaptação transcultural de instrumentos de avaliação psicológica: Levantamento dos estudos realizados no Brasil de 2000 a 2010. Psico-USF, 16(3), 367-381.doi: 10.1590/ S1413-82712011000300013

Ritzler, B. (2004). Cultural applications of the Rorschach, apperception tests and figure drawing. Em M. Hilsenroth \& D. Segal (Eds.). Personality Assessment. vol. 2. Comprehensive Handbook of Psychological Assessment (pp. 315-342). Hoboken, NJ: John Wiley and Sons.

Vilches, L., \& Olivos, S. (2004). Propuesta de parâmetros referenciales para la utilizacion del Test del Zulliger individual em seleción del personal. Memória para optar al título de psicólogo. Santiago del Chile: Universidad del Chile.

Villemor-Amaral, A. E., \& Cardoso, L. M. (2012). Validade convergente do tipo de vivência (EB) no teste de Zulliger/SC. PSICO, 43(1), 109-115. Recuperado de http://revistaseletronicas.pucrs.br/ojs/index. $\mathrm{php} /$ revistapsico/article/viewFile/11105/7625

Villemor-Amaral, A. E., \& Pasqualini-Casado, L. (2006). A cientificidade das técnicas projetivas em debate. Psico-USF, 11(2), 185-193. Recuperado de http:// www.scielo.br/pdf/pusf/v11n2/v11n2a07.pdf

Villemor-Amaral, A. E., \& Primi, R. (2012). Teste de Zulliger no Sistema Compreensivo ZSC: Forma individual. São Paulo: Casa do Psicólogo.

Zdunic, A. L. (2003). El teste Zulliger en la evaluación psicológica de personal. Aportes del sistema compreensivo de Exner. Cuadernos de Evaluación Psicológica (2 $2^{\text {a }}$ ed). Buenos Aires: Paidós.

Zdunic, A. L. (2011). Zulliger-500. Actualizando datos normativos. Memorias do III Congresso Internacional de Investigación y Práctica profesional en Psicología. XVIII Jornadas de Investigación y Séptimo Encuentro de Investigadores en Psicología del Mercosur (pp. 143-144). Buenos Aires, Argentina.

Weiner, I. B. (2000). Princípios da interpretação do Rorschach. São Paulo: Casa do Psicólogo.

World Trade Organization (2012). Recuperado de http://www.wto.org

Recebido em: 15/08/2014 Primeira reformulação em: 18/02/2015 Segunda reformulação em: 14/04/2015 Aprovado em: 24/07/2015 
Sobre as autoras:

Jucelaine Bier Di Domenico Grazziotin é mestre em Envelhecimento Humano e especialista em Avaliação Psicológica e em Gestão Empresarial, pela Universidade de Passo Fundo (UPF-RS).

E-mail: jucelainegraz@terra.com.br

Silvana Alba Scortegagna é doutora em Psicologia pela Universidade São Francisco/Itatiba (USF-SP) e docente do Programade Pós-Graduação em Envelhecimento Humano da Universidade de Passo Fundo (UPF-RS).

E-mail:silvanalba@upf.br

Contato com as autoras:

Silvana Alba Scortegagna

Programa de Pós-Graduação Stricto Sensu em Envelhecimento Humano

Universidade de Passo Fundo

BR 285, Km 171, Bairro São José

Cx. Postal 611 - Passo Fundo/RS - Brasil

CEP: 99052-900

E-mail:silvanalba@upf.br 